Reprod. Nutr. Dévelop., 1980, 20 (6), 1899-1905.

\title{
Survie des gamètes de truite Arc-en-ciel après dilution dans des solutions salines ou de sucrose
}

par R. BILLARD

Laboratoire de Physiologie des Poissons, I. N. R. A. 78350 jouy en josas.

Summary. Survival of rainbow trout gametes after dilution in mineral or sucrose solutions.

Ova or spermatozoa of the rainbow trout, Salmo gairdneri, were exposed during increasing time lengths to sucrose solutions (200-250 and $300 \mathrm{mOsm}$ ) and to a medium reconstituting the mineral composition of the seminal fluid of this species (MMLS). Treatment results were evaluated by the number of eyed-eggs after 20 days of incubation at $10^{\circ} \mathrm{C}$. After $1 / 100$ and $1 / 1000$ dilution in various media, the spermatozoa did not move and their fertilizing ability was entirely maintained for $40 \mathrm{~min}$ (1/1000 dilution) or $60 \mathrm{~min}$ (1/100 dilution). Egg survival was brief in the sucrose media $(<20 \mathrm{~min})$, but better in the MMLS, at least in one experiment (fig. 4). These media can be used to dilute trout sperm either for short-term storage or for deep-freezing, but mineral elements should be added.

\section{Infroduction.}

Les spermatozoìdes de salmonidés sont immobiles dans le tractus génital ou dans le sperme et ne sont mis en mouvement qu'après dilution dans de l'eau douce (Huxley, 1930 ; Billard, 1978) ou dans des solutions physiologiques dont la salinité n'excède pas 10-12 p. 1000 (Nomura, 1964 ; Billard et al., 1974). La durée de motilité des spermatozoïdes est toujours brève ( $30 \mathrm{sec}$ à quelques $\mathrm{min}$ ) et le pouvoir fécondant ne se maintient à son niveau initial que pendant 15 à $30 \mathrm{sec}$ pour devenir nul après 1 à $2 \mathrm{~min}$ (Smirnov, 1963 ; Ginsburg, 1968 ; Billard ef al., 1974). Cela signifie-que les spermatozoïdes doivent, dès leur mise en mouvement, se trouver en présence des ovules. Cependant, il est parfois nécessaire de procéder à une dilution du sperme en vue de conservation ou de congélation et il est alors impératif de prévenir la mise en mouvement des spermatozoïdes. Pour cela, on utilise généralement un dilueur enrichi en $\mathrm{K}^{+}$(Billard et Jalabert, 1974). C'est en effet la présence de $\mathrm{K}^{+}$dans le liquide séminal qui inhibe la mobilité des spermatozoïdes de salmonidés (Schlenk et Kahmann, 1937). $\mathrm{Si}$ des dilueurs enrichis en $\mathrm{K}^{+}$permettent la dilution en vue d'une conservation des spermatozoïdes à court terme (Billard, 1975), ils ne sont pas favorables à la congélation car après décongélation on observe des motilités spontanées (Billard, non publié). Un autre produit, le sucrose qui a la propriété d'inhiber la motilité des spermatozoïdes a récemment été proposé pour diluer le sperme de salmonidés en vue de la congélation (Mounib, 1978 ; Legendre, 1979). 
Le présent travail porte sur la comparaison des effets de solutions de sucrose et de solutions minérales sur la survie des gamètes de truite arc-en-ciel en retenant comme critère la conservation du pouvoir fécondant des spermatozoïdes dont la motilité a été inhibée et le maintien de la fertilité des ovules dilués.

\section{Matériel et méthode.}

Les géniteurs de truite arc-en-ciel Salmo gairdneri (souche « Lubin », âgés de 2 ans pour les mâles et 3 ans pour les femelles) sont élevés dans la pisciculture de Gournay-sur-Aronde et introduits au laboratoire en fin de gamétogenèse (nov. 1978). L'expérience a lieu un mois environ après le début de la spermiation et les ovules sont prélevés sur des femelles ayant ovulé depuis moins de 1 semaine. Le principe expérimental consiste à exposer le sperme ou les ovules aux différents dilueurs à tester pendant des temps croissants, puis après rinçage à procéder à l'insémination avec du sperme ou des ovules frais. L'effet du traitement est apprécié par le taux de développement embryonnaire. Les gamètes sont prélevés sur au moins 5 géniteurs différents et mis en pool. La motilité est vérifiée au microscope $(\times 100)$ pour chaque mâle, les spermes peu motiles sont éliminés. Les pontes présentant des ovules surmatures ou ayant des quantités excessives de liquide coelomique sont rejetées.

Trois milieux à base de sucrose (Prolabo RP) ajustés à une pression osmotique (p.o.) de $300\left(S_{300}\right), 250\left(S_{250}\right)$ et $200\left(S_{200}\right)$ milliosmoles et tamponnés à pH 9 (Tris $0,02 \mathrm{M}$ ) sont comparés au milieu minéral du liquide séminal (MMLS) p.o 250 milliosmoles, pH 9 (tampon Tris 0,02 M) (Billard et Jalabert, 1974) et dans certains cas au dilueur d'insémination artificielle (DIA) p.o. 250 milliosmoles $\mathrm{pH} 9$ (Tris $0,02 \mathrm{M}$, glycocolle $0,05 \mathrm{M}$ ).

Conservation des spermatozoïdes. - Du sperme est dilué au $1 / 100\left(10^{-2}\right)$ et au $1 / 1000\left(10^{-3}\right)$ dans les milieux $S_{300}, S_{250}, S_{200}$ et MMLS pendant 20,40 et $60 \mathrm{~min}$ (Exp. 1) et 40,80 et $160 \mathrm{~min}$ (Exp. 2), à la température de $10^{\circ} \mathrm{C}$. Le mélange sperme-dilueur est ensuite centrifugé $(1500 \mathrm{~g}, 5 \mathrm{~min})$, le surnageant est éliminé et remplacé par du MMLS dont on sait qu'il est bien toléré par les ovules. Les spermatozoïdes sont remis en suspension dans le MMLS et l'insémination est pratiquée immédiatement après selon la fechnique de double dilution (Billard et Jalabert, 1974) : $10 \mathrm{ml}$ du mélange spermatozoïdes + MMLS sont ajoutés à un lot d'environ 200 ovules dilués dans $10 \mathrm{ml}$ de DIA. Dans ces conditions, le taux de potassium est abaissé, les spermatozoïdes sont mis en mouvement et la fécondation peut avoir lieu.

Au temps $0\left(t_{0}\right)$ le sperme est mélangé aux mêmes milieux et immédiatement centrifugé. Une insémination témoin est pratiquée d'autre part directement dans le DIA. Les expériences 1 ef 2 ont été réalisées avec des pools de sperme et d'ovules différents. Dans une expérience complémentaire, les milieux à base de sucrose auxquels le sperme a été exposé pendant $40 \mathrm{~min}\left(10^{-3}\right)$ n'ont pas été éliminés après centrifugation et ont servi à inséminer directement des ovules préalablement mélangés à $10 \mathrm{ml}$ de DIA.

Conservation des ovules. - Les ovules prélevés sur plusieurs femelles sont soigneusement mélangés et égouttés afin d'éliminer le liquide coelomique, puis répartis 
par lots d'environ 200 dans de petites coupelles. On y ajoute $10 \mathrm{ml}$ de chacun des dilueurs à tester soit $S_{300}, S_{250}, S_{200}, M M L S$ et DIA. Les durées d'exposition des ovules à ces différents dilueurs sont de 20 et $40 \mathrm{~min}$ (Exp. 3) ef 40,80 ef $160 \mathrm{~min}$ (Exp. 4). Les milieux sont alors éliminés par passage des ovules sur un tamis ef remplacés par $10 \mathrm{ml}$ de DIA dans lequel l'insémination est immédiatement pratiquée avec 10 ou $100 \mu l$ de sperme frais (dilution $10^{-3}$ ou $10^{-2}$ ). $\mathrm{A}_{\mathbf{0}}$ les différents dilueurs sont mélangés aux ovules pendant quelques secondes puis remplacés par du DIA, milieu dans lequel l'insémination est pratiquée. Les expériences 3 ef 4 font appel à des pools de sperme et d'ovules différents. Après insémination, les ovules sont laissés dans le même milieu pendant $16 \mathrm{~min}$, puis chaque lot est transféré en eau douce dans des incubateurs compartimentés et thermorégulés à $10^{\circ} \mathrm{C}$ dans lesquels le développement embryonnaire a lieu sous obscurité ou en lumière atténuée. Après 20 jours d'incubation les œufs sont fixés au liquide de Stockard et le dénombrement porte sur les œufs embryonnés normaux, anormaux et avortés, ainsi que sur les cufs non fécondés.

Les résultats sont analysés, par test $\chi^{2}$ (comparaison de deux pourcentages de fécondation) ou par analyse de variance (comparaison de traitements), après transformation angulaire des valeurs.

\section{Résultats.}

Conservation du sperme ò l'état dilué. - Les spermatozoïdes, examinés au microscope, demeurent immobiles après dilution dans les milieux $S$ et MMLS et ne sont mis en mouvement qu'après une seconde dilution dans le DIA.

Un effet de la p.o. se manifeste après une durée d'exposition de $60 \mathrm{~min}$ (fig. 1) ; le pouvoir fécondant du sperme dilué au 1/1 000 dans $S_{250}$ ef MMLS est significativement $(P<0,05)$ plus élevé que celui dilué dans $S_{300}$ et $S_{200}$.

Après 20 ef $40 \mathrm{~min}$ d'exposition il n'y a pas de différence significative à la fois entre les différents milieux ef par rapport à $t_{0}$. Dans la seconde expérience (fig. 2 ) on retrouve pour le même taux de dilution (1/100) la supériorité du MMLS mais $S_{300}$ se révèle ici légèrement plus favorable à $S_{250}$.

Un effet du taux de dilution apparaît en outre sur la figure 2 où le pouvoir fécondant est nettement et significativement $(P<0,01)$ plus élevé à $10^{-2}$ qu'à 10-3. Par contre dans l'expérience 1 il n'y a pas de différence entre la dilution de $10^{-3}$ (voir fig. 1) et celle de $10^{-2}$.

Lorsque les milieux sucrosés ne sont pas remplacés par le MMLS ef lorsqu'ils sont directement appliqués sur les ovules, les résultats ne sont pas modifiés.

Conservation des ovules après dilution. - L'aptitude à la fécondation des ovules est considérablement ef significativement diminuée $(P<0,001)$ après 20 ef 40 min d'exposition à fous les milieux festés (Exp. 3, fig. 3). La chute de fertilité est cependant moins marquée avec MMLS qu'avec les milieux sucrosés. Parmi ceux-ci, $S_{250}$ est légèrement moins délétère que les autres, mais les différences ne sont pas significatives. Pour les durées d'exposition plus longues (Exp. 4, fig. 4), le caractère toxique des milieux à base de sucrose se retrouve avec des fécondabilités nulles à la $40^{\mathrm{e}} \mathrm{min}$, alors qu'elles sont intégralement conservées après $160 \mathrm{~min}$ de séjour dans le MMLS ef le DIA. Aucune anomalie de développement embryonnaire n'a été détectée. 


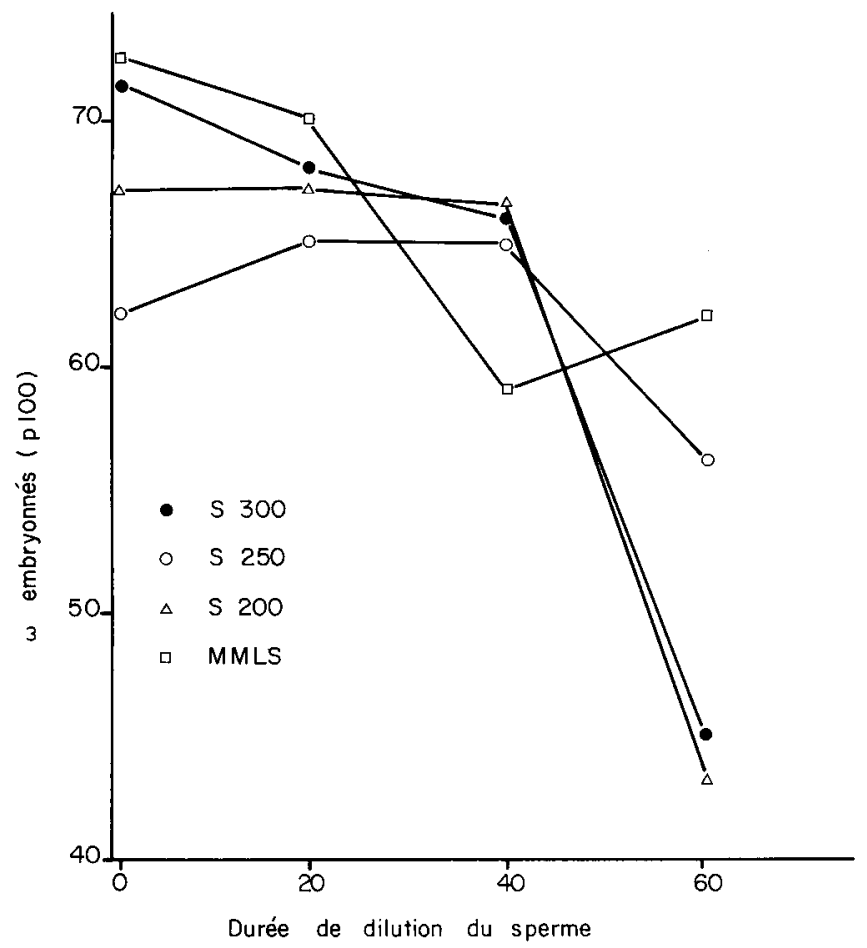

FIG. 1. - Survie du sperme de truite Arc-en-ciel dilué au $1 / 1000$ dans des solutions de sucrose $S(200,250$ ef 300 mosmoles) ef dans MMLS, puis exposé à ces solutions pendant des temps croissants : 0,2040 , $60 \mathrm{~min}$. Dilution finale lors de l'insémination $0,5.10^{-3}$ soit $4,05.10^{6} \mathrm{spermatozoïdes} / \mathrm{m} /$ de dilueur. Taux de fécondation de l'insémination témoin pratiquée directement dans le DIA : 68 p. 100.

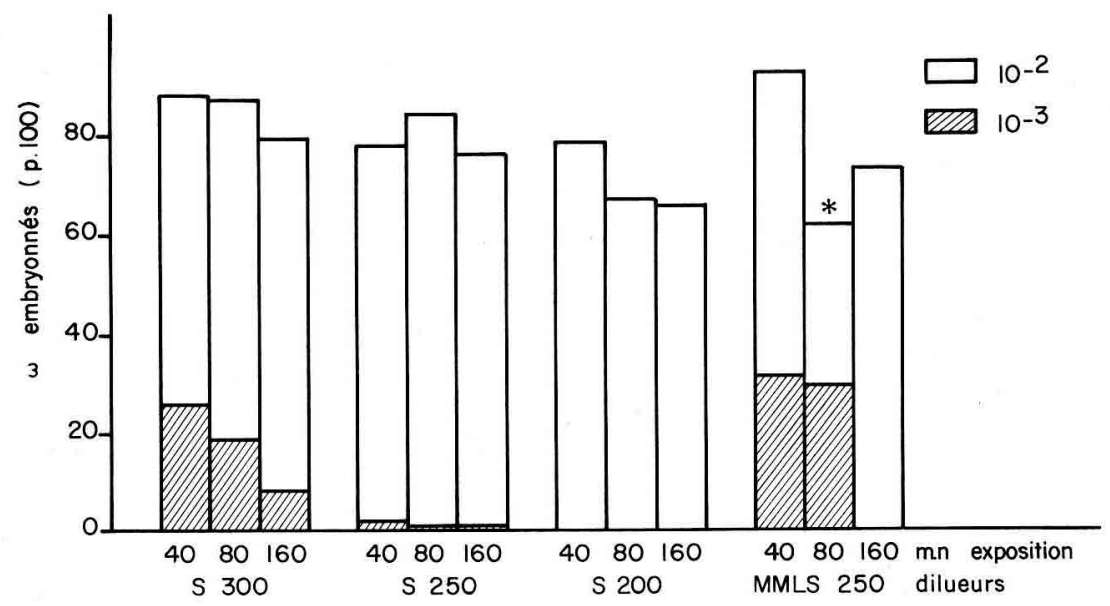

FIG. 2. - Survie du sperme de truite Arc-en-ciel dilué dans des solutions de sucrose ef dans MMLS (taux de dilution $10^{-2}$ ef $10^{-2}$ ) ef exposé pendant 40,60 ef $160 \mathrm{~min}$. Dilution finale du sperme lors de l'insémination : $0,5 \cdot 10^{-2}\left(62,5.10^{6} \mathrm{spz} / \mathrm{ml}\right.$ de $\left.D I A\right)$ et $0,5.10^{-3}\left(6,2510^{6} \mathrm{spz} / \mathrm{ml}\right.$ de DIA). Taux de fécondation de l'insémination témoin : 79 p. 100 à $0,5 \cdot 10^{-2}$ et 41 p. 100 à $0,5 \cdot 10^{-3} .^{*}: P<0,05$. 


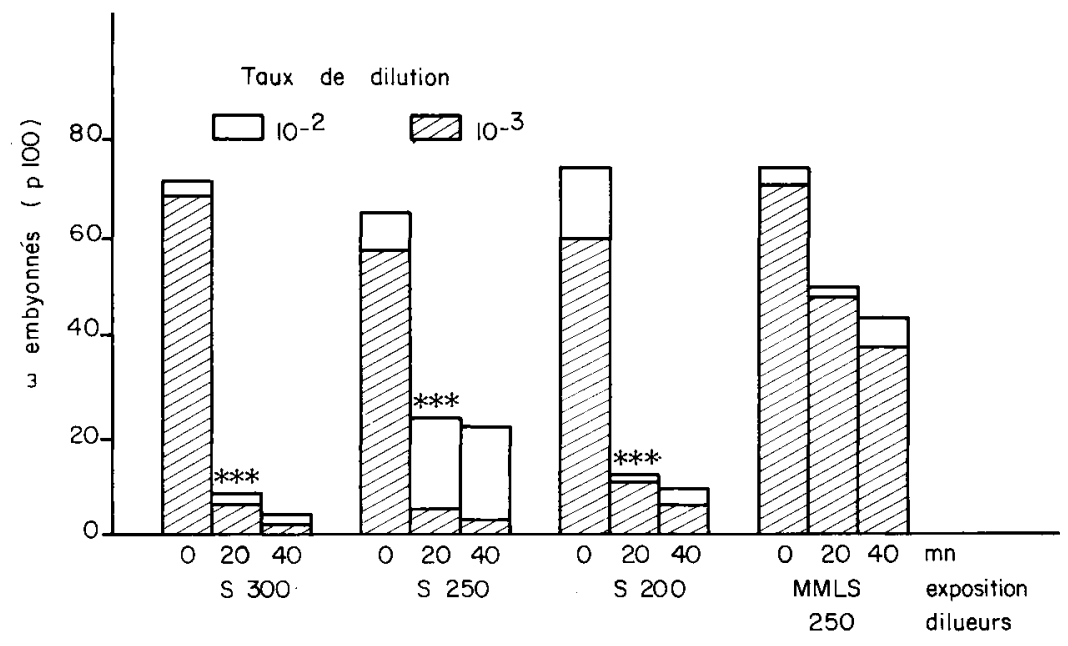

FIG. 3. - Survie des ovules de truite Arc-en-ciel après dilution dans des solutions de sucrose ef du MMLS ef exposition pendant 20 et $40 \mathrm{~min}$ ( 200 ovules environ pour $10 \mathrm{ml}$ de milieu). Lors de l'insémination le DIA est substifué aux milieux d'exposition. Dilution du sperme $10^{-2}\left(65.10^{6} \mathrm{spz} / \mathrm{ml}\right.$ de DIA) ef $10^{-3}\left(6,5.10^{6} \mathrm{spz} / \mathrm{ml}\right.$ de DIA). Taux de la fécondation témoin pratiquée dans le DIA : $81 \mathrm{p} .100$ à $10^{-2}$ et 82 p. 100 à 10-3.*** : différence hautement significative $(P<0,001)$ par rapport à $t_{0}$.

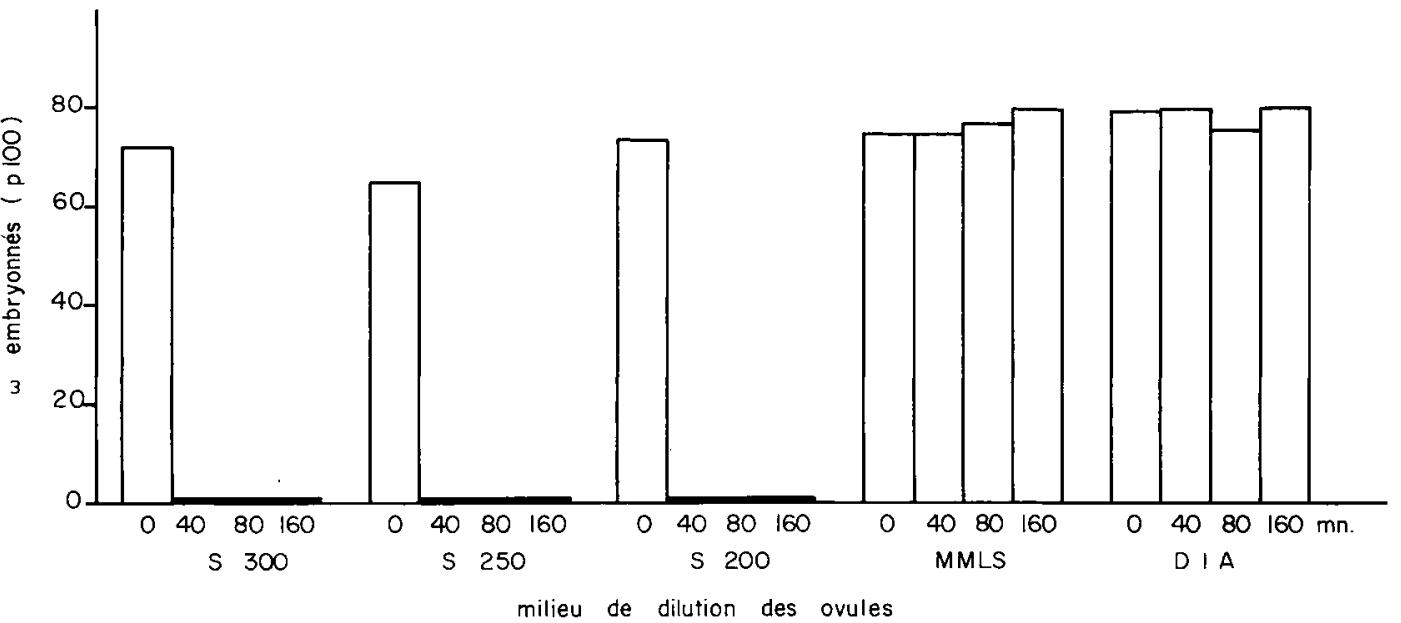

FIG. 4. - Survie des ovules dans des solutions de sucrose, du MMLS et du DIA pendant 40,80 et $160 \mathrm{~min}$. Dilution du sperme lors de l'insémination $10^{-3}\left(8,4.10^{6} \mathrm{spz} / \mathrm{ml}\right.$ de DIA $)$.

\section{Discussion.}

Les milieux à base de sucrose autorisent des possibilités de dilution du sperme de truite sensiblement comparables à celles du MMLS, mais se révèlent extrêmement défavorables à la survie des ovules. Cependant, ces derniers supportent sans dommage 
la technique d'insémination par double dilution, c'est-à-dire apport de sperme dilué en milieu sucrosé sur des ovules préalablement dilués dans du DIA. Il est dès lors possible d'introduire du sucrose dans les milieux de dilution pour sperme de salmonités et cela est particulièrement indiqué dans le cas des dilueurs de congélation.

En effet, avec les dilueurs enrichis en $\mathrm{K}^{+}$habituellement utilisés, on observe à la décongélation des motilités spontanées qui ne se produisent pas après emploi de solutions comportant du sucrose (Billard, non publié). Les résultats des présentes expériences montrent que ces milieux peuvent être employés lors de l'insémination à condition qu'ils ne soient pas mis directement au contact des ovules aux concentrations testées et que la durée de séjour ne soit pas prolongée. Ces conditions sont réunies dans le cas d'insémination avec du sperme congelé qui implique obligatoirement la technique de double dilution ef dans laquelle le contact des ovules avec le milieu de dilution du sperme ne dépasse pas $15 \mathrm{~min}$. En outre, dans le cas de la congélation du sperme de salmonidés, le taux de dilution avec les milieux de congélation est faible (1 vol. sperme/3 vol. dilueur ; Legendre et Billard, 1980), de sorte qu'après décongélation la quantité de sucrose mise en contact avec les ovules sera réduite.

L'importance de la p.o. du dilueur sur le pouvoir fécondant du sperme a déjà été démontrée (Petit et al., 1973). Dans le cas d'une solution saline comme le DIA, la p.o. la plus favorable est de 250 milliosmoles (Billard ef al., 1974). Avec le sucrose elle est de 250 dans l'expérience 1 et de 300 dans l'expérience 2. Ces différences peuvent être attribuées à une grande hétérogénéité dans la qualité des gamètes qui se rencontre fréquemment chez les salmonidés (Carpentier et Billard, 1978).

A l'effet de la pression osmotique s'ajoute un effet ionique incontestable comme le montrent la supériorifé du MMLS par rapport aux milieux $S$ (exclusivement organiques) et l'effet néfaste des fortes dilutions. Cet effet ionique se retrouve de façon encore plus marquée dans le cas de la dilution des ovules où la survie se conserve intégralement pendant 160 min dans les milieux minéraux (DIA, MMLS) alors qu'elle est déjà nulle après 40 min de dilution dans les milieux $S$ (fig. 4). Il apparaît donc que les dilueurs pour gamètes de salmonidés doivent réunir des conditions de p.o. et de composition ionique bien définies.

En conclusion, des milieux contenant du sucrose ef développant des pressions osmotiques de 250 à 300 milliosmoles peuvent être utilisés pour diluer du sperme de truite sans entrainer la mise en mouvement des spermatozoïdes et sans modifier notablement le pouvoir fécondant. Ces milieux sucrosés additionnés des éléments minéraux entrant dans la composition du liquide séminal constituent donc un excellent dilueur de conservation et de congélation.

Reçu en avril 1980.

Accepté en juillet 1980.

Remerciements. - Ce travail a été pris en charge par le Conseil Supérieur de la Pêche. Nous remercions MM. Levillain, Le Brenn et Puype pour les soins apportés aux animaux et $M$. Marie pour le contrôle de la qualité des eaux de la pisciculture et de la salle d'incubation. La présentation dactylographique du manuscrit est due à Catherine Clappier. 


\section{Références}

BILLARD R., 1975. L'insémination artificielle de la truite Salmo gairdneri Richardson. IV. Effets des ions $\mathrm{K}^{+}$ef $\mathrm{Na}^{+}$sur la conservation du pouvoir fécondant des gamètes. Bull. Fr. Pisc., 256, 88-100.

BILLARD R., 1977. Utilisation d'un système Tris-glycocolle pour tamponner le dilueur d'insémination de truite. Bull. Fr. pisc., 264, 102-112.

BILLARD R., 1978. Changes in structure and fertilizing ability of marine and freshwater fish spermatozoa diluted in media of various salinities. Aquaculture, 14, 187-198.

BILLARD R., JALABERT B., 1974. L'insémination artificielle de la truite Salmo gairdneri Richardson. II. Comparaison des effets de différents dilueurs sur la conservation de la fertilité des gamètes avant et après insémination. Ann. Biol. anim. Bioch. Biophys., 14, 601-610.

BILLARD R., PETIT J., JALABERT B., SZÖLLÖSI D., 1974. Artificial insemination in trout using a sperm diluant, 715-723. In BLAXTER J. H. S., Early life history in fish. Springer Verlag, Berlin, Heidelberg, New York.

CARPENTIER P., BILLARD R., 1978. Conservation à court terme des gamètes de salmonidés à des températures voisines de $0^{\circ} \mathrm{C}$. Ann. Biol. anim. Bioch. Biophys., 18, 1083-1088.

GINSBURG A. S., 1968. Ferfilization in fishes and the problems of polyspermy. Akad. Nauk. USSR, NOAA and Nat. Sci. Found Transl., 290 pp.

HUXLEY J. S., 1930. The maladaptation of trout spermatozoa to freshwater. Nature, 125, 494.

LEGENDRE M., 1979. Contribution d̀ l'étude de la congélation du sperme chez lo truite Arc-en-ciel (Salmo gairdneri). DEA, Univ. Paris VI, 20 pp.

LEGENDRE M., BILLARD R., 1980. Cryopreservation of rainbow trout sperm by deep freezing. Reprod. Nutr. Develop., 20, 1859-1868.

MOUNIB M. S., 1978. Cryogenic preservation of fish and mammalian spermatozoa. J. Reprod. Ferf., 53, $13-18$.

NOMURA M., 1964. Studies on reproduction of rainbow trout, Salmo gairdneri with special reference to egg taken. VI. The activities of spermatozoa in different diluents, and preservation of semen. Bull. jap. Soc. Sci. Fish, 30, 723-733.

PETIT J., JALABERT B., CHEVASSUS B., BILLARD R., 1973. L'insémination artificielle de la truite Salmo gairdneri Richardson. I. Effets du taux de dilution, du $\mathrm{pH}$ et de la pression osmotique du dilueur sur la fécondation. Ann. Hydrobiol., 4, 201-210.

SCHLENK W., KAHMANN H., 1937. Die chemische Zusammensetzung des Spermaliquors und ihre physiologische Bedeutung. Biochem. Z., 295, 283-301.

SMIRNOV A. I., 1963. Conservation dans l'eau de l'aptitude des œufs ef des spermatozoïdes du saumon Oncorhynchus gorbuscha W. à la fécondation. Nauchn. Dokl. Wyssh. Shk. biol. Nauki SSSR, 3, 37-41. 